\title{
Model-based Verification and Optimization of Batteries for Mobile Power Applications
}

\author{
Marco Franke, Tamas Juhasz, Ulrich Schmucker \\ Fraunhofer Institute for Factory Operation and Automation IFF \\ Sandtorstr. 22, 39106 Magdeburg, Germany \\ \{marco.franke,tamas.juhasz,ulrich.schmucker\}@iff.fraunhofer.de
}

\begin{abstract}
This paper describes a new approach to sizing an electric drivetrain, including its power supply. The advantages of a real battery testing system are combined with the advantages of a Modelica model of a product. A testing system analyzes battery performance under specific constraints such as differing temperatures, vibrations and humidity. Since these constraints are hard to replicate in a model, an experimental rig is an optimal solution for battery tests [1]. Virtual engineering of a real system is advantageous in terms of the speed of modifications, the measurement of all relevant data and the low cost of development. The coupled virtual model and experimental testing rig for batteries constitute the basis for this new concept and improve the development of the electric powertrain.
\end{abstract}

Keywords: battery, hardware-in-the-loop, real-time

\section{Introduction}

Mobile applications are increasingly using electric power supplies, which have to be sized optimally. Electric vehicles are stimulating the development and optimization of future drivetrains. Their physical and temporal range is very important.

This study focuses on one of the main components, the power supply. Batteries are normally tested in test rig environments. The advantages of an experimental rig are its versatility, e.g. for climate tests, vibration tests and discharge tests. One of its most important features its safety for operators and the environment. Tests of batteries installed in real vehicles or equipment involve various safety issues and unforeseen conditions or defects can cause injuries or other safety-critical situations.

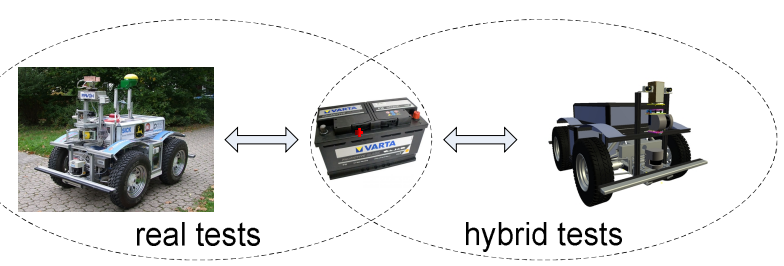

Figure 1: Hardware-in-the-loop with a real battery

Since current battery models are often incomplete or only describe properties in certain areas, the advantages and potentials of real test environments also ought to be factored into the modeling of mobile systems and, specifically into the modeling of electric drivetrains.

\section{The Experimental Vehicle RAVON}

Since detailed data from vehicle manufactures was unavailable for the development of the methodology presented here, the AWD off-road vehicle RAVON (Robust Autonomous Vehicle for Off-road Navigation) developed by the Technical University of Kaiserslautern, Germany was used as the test subject.



Figure 2: The autonomous vehicle RAVON 
This unmanned electric vehicle is normally used as a test object to study autonomous, behavior-based strategies for motion adaptation, localization and navigation in rough outdoor terrain. Equipped with four synchronous wheel hub motors and four batteries, the vehicle can be used under a variety of temperature, humidity and terrain conditions. The electric vehicle is powered by eight $12 \mathrm{~V}$ batteries with a total voltage of $48 \mathrm{~V}$. This power supply must be adequate for the entire task.

Since the data needed to create a mechatronic model of RAVON (e.g. CAD data, drivetrain and control parameters and battery specifications) were available in advance, modeling with Modelica could start immediately.

\section{The Modeling Procedure}

The RAVON's CAD data are available in STEP format. These data are the basis for the mechanical parts of the chassis with interfaces to other physical domains in Modelica. The Fraunhofer Institute for Factory Operation and Automation IFF in Magdeburg has an automated CAD-to-simulation procedure that effectively converts CAD data into Modelica description [2].

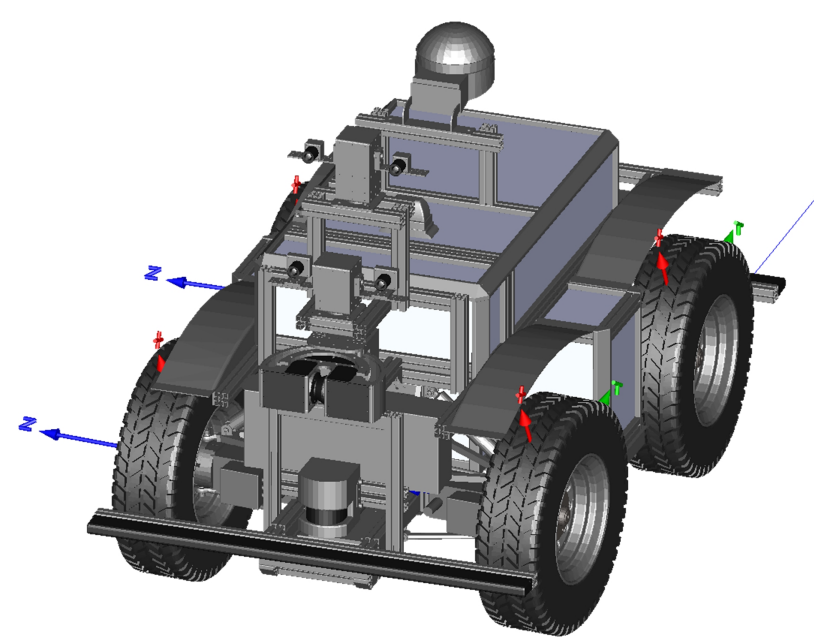

Figure 3: Modelica model generated semiautomatically from CAD data

The program's database contains XML data on the mechanical model's physical and kinematic properties (masses, moments of inertia, joint constraints, etc.) as well as triangulated VRML geometry exported directly from the CAD system. Since it also supports closed kinematic loops, this new procedure also makes it possible to model any complex mechanical system efficiently.

\subsection{RAVON Subsystems}

The virtual RAVON platform must communicate with a real battery test rig in real time. Apart from incorporating the semi-automatically generated chassis model, the most demanding task was modeling the entire electric drivetrain so that it is real-time capable. The uppermost vehicle model was decomposed into two Modelica subsystems, Chassis and Drive (see Figure 4).

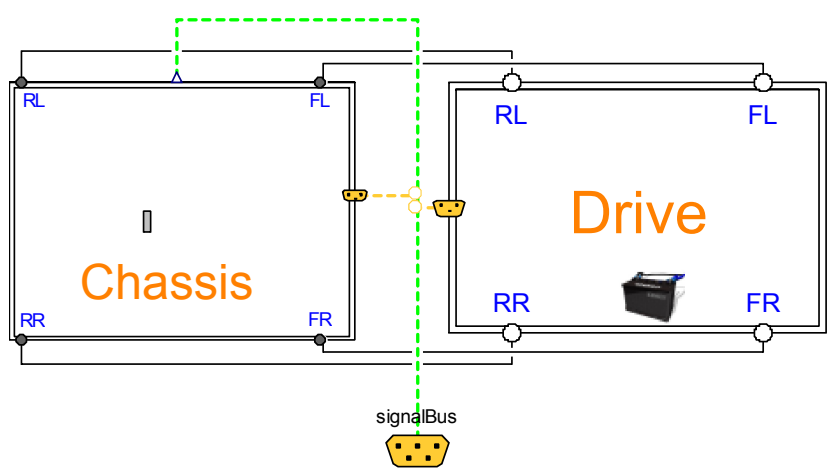

Figure 4: Modeled subsystems of RAVON

The virtual parts of the Drive model can be matched with their real counterparts. A Huebner HAC 71.11.6 permanent magnet synchronous induction machine and a CycloDrive 6000 planetary gear are mounted in each wheel-hub model. The electrical machines were parameterized in Modelica using real manufacturer data. A DC interface to a common external power supply was also included.

The drive subsystem of a single wheel with the $\mathrm{DC} / \mathrm{AC}$ converter and the motor controller is pictured in Figure 5.

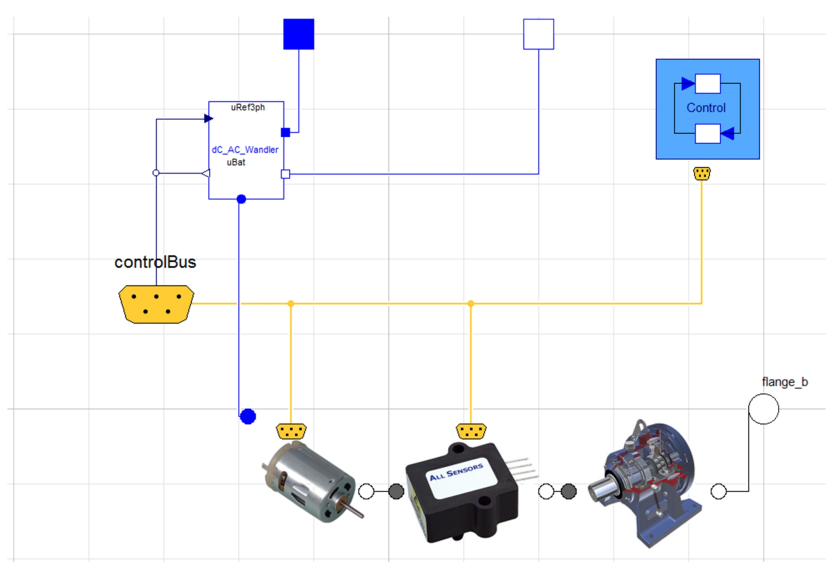

Figure 5: Controlled wheel-hub motor model 
The motor controller model is based on a conventional cascade structure with an outer speed control loop and an inner current control loop. PI controllers with limited outputs are used in both cases. Since the real-time capability had top priority, some simplifications were made in the model of the motors' power electronics, among others. Since they would not influence the model's accuracy significantly, fast PWM signals were not included. The torque control algorithm is based on field-oriented vector control [3]. The controller parameters were selected to attain maximum speed with minimal overshoot.

\section{Battery Testing System}

This study aimed to utilize a real battery testing system in order to integrate a real battery's complex behavior in early stages of product development. Rather than using a real product prototype to record battery charging profiles, a virtual prototype is incorporated in the workflow. A real testing rig can be used to swap energy bidirectionally between the virtual model and the real battery, depending on the prevailing conditions.

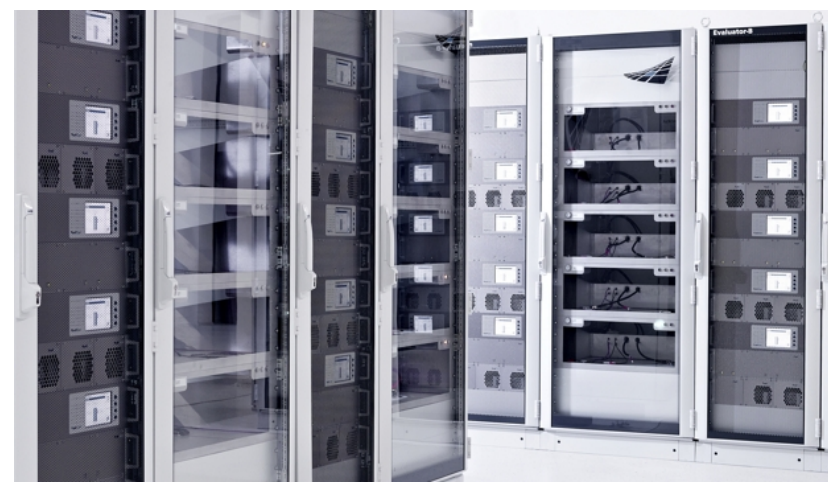

Figure 6: FuelCon battery testing system

Since the Modelica model provides the load for a real battery, its performance can be analyzed dynamically. The testing system from the company FuelCon allows users to set different environmental parameters such as ambient pressure, temperature, atmospheric humidity and movement. Various types of batteries, e.g. lithium-ion, lithium-polymer, NiCd, $\mathrm{NiMH}$ or lead-acid, can be tested with this testing system. The Evaluator-B series battery testing system has an interface that is connected with external current profiles [4]. The methodology developed here with the virtual vehicle model uses this function to repeatedly supply actual current and receive the battery voltage.

\section{Integration of the Real Testing Sys- tem in a Hybrid Simulation}

The objective of this study was to integrate a real battery testing environment in a hardware-in-theloop simulation setting. Battery voltage and computed electric current signals must be exchanged bidirectionally during a test run. The testing system is equipped with load and charger electronics. This makes it possible to charge or discharge the batteries depending on prevailing electric currents. Dynamic processes such as current peaks can also be analyzed with this approach.

\subsection{Software Interfaces}

The FuelCon testing system's operator interface utilizes Visual Basic (VB) scripts running in an embedded Windows environment. The Fraunhofer IFF developed the COMsigate interface to extend the operator interface's range of functions. The COMsigate software module employs the User Datagram Protocol (UDP) for communication between the testing system and the outside world.

The main VB script runs in a loop during batterycoupled hardware-in-the-loop tests. It reads the actual battery voltage $(U)$ and can set a new electric load (I) every $10 \mathrm{~ms}$ in keeping with the latest UDP packet received through the COMsigate.

Matlab/Simulink was incorporated in the tool chain because of its excellent interoperability and advanced toolboxes [5]. Its UDP communication blocks were used to transmit the battery signals $U$ and $I$ bidirectionally through the COMsigate interface. In addition autonomous driving course profiles, the vehicle model can also be interactively controlled with a joystick module that enables users to define own test cycles in Matlab.

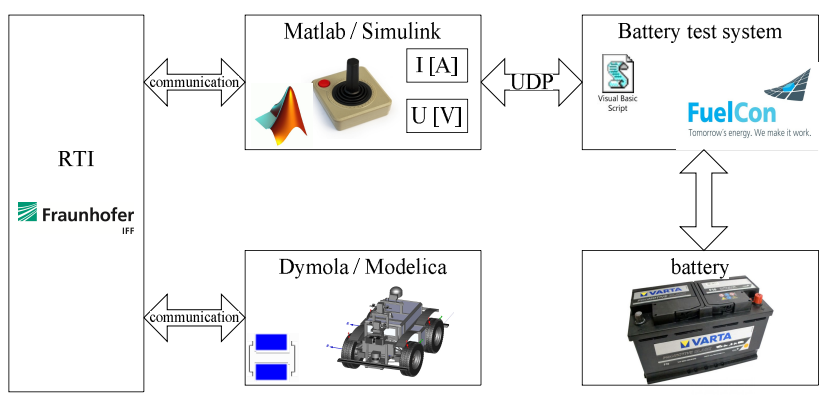

Figure 7: Tool chain of the hybrid simulation of RAVON with a real battery in a test rig 
The Fraunhofer IFF developed the Real Time Interface (RTI) to support real-time communication between heterogeneous software applications [6]. RTI's shared memory is used to exchange battery charge states and reference speed signals between Matlab/Simulink and the Dymola simulator [7] running the RAVON model.

The reference vehicle speed and the axles' steering movement are supplied to Dymola. Each electric drive is controlled separately. The vehicle's acceleration is determined by the electric current in the respective motors. The virtual supply voltage is the value of the real battery measured in the test rig. Thus, the real battery's performance influences the simulated vehicle's electric power.

\section{Results}

The first tests revealed that the dynamic processes between the battery testing system and the vehicle model reflect the theoretical considerations. The first example (Figure 8) presents a battery performance test using a static power consumption scenario. The model vehicle had to travel at a constant reference speed. Decreasing voltage increases the demand for electric current as the battery discharge. Since the mechanical power demanded is constant this phenomenon can be explained by the electric power balance equation: $P_{\text {elec }}=U \cdot I$.


Figure 8: Discharging at constant travel speed

The actual speed remains constant until the maximum current is reached, which is defined by the battery. From this point onward, the vehicle's speed cannot be kept constant and decreases. Afterward, the electric current reaches a constant value and the battery voltage continues to fall.

The results presented in Figure 9 depict forward and backward acceleration and deceleration maneuvers with sudden stops. The battery's demand for current increases during the acceleration phase and the battery's voltage drops temporarily. The current peak when the electric motor switches from loading to braking is evident. Such current peaks during charging and discharging can affect a battery's life and also influence a battery's state of charge.
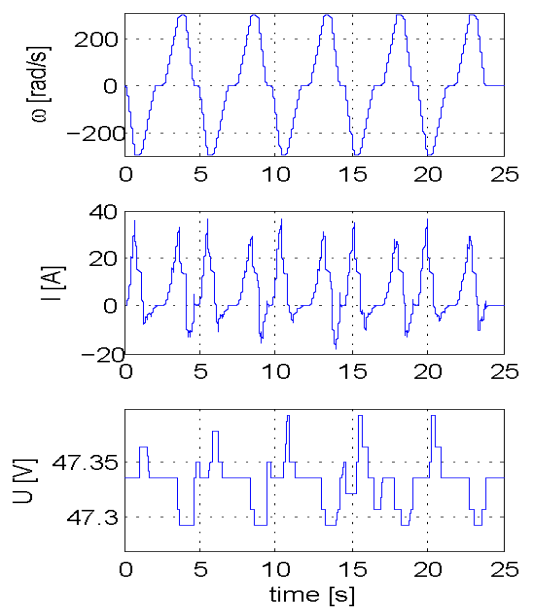

Figure 9: Analysis of dynamic behavior

A continuous and rapid change of direction was investigated in the next test scenario. Electric current and voltage propagation were analyzed. Figure shows the rate of speed as well as the related current and voltage.
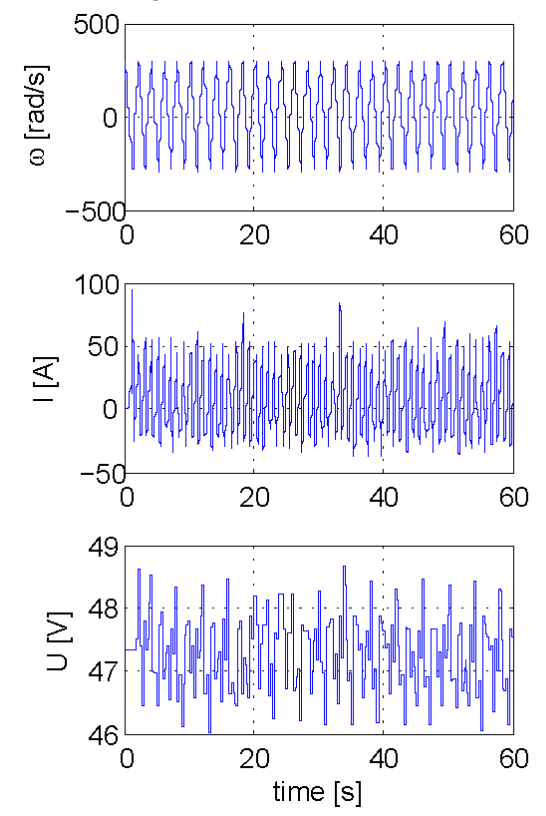

Figure 10: Continuous and rapid changes of direction 
The current peaks in the case presented here are many times higher than those in the test scenario in Figure 9.

\section{Conclusions}

This paper describes a novel hardware-in-the-loop approach to connecting mobile mechatronic system models with a real battery testing system. It can be used to test the entire system's energy balance under realistic conditions. This makes it possible to perform stress and durability tests without having to build complex prototypes first. Battery size and the product's electromechanical properties can be optimized. As a result, product development is expedited and validated by early testing. This approach is suitable for more than just electric vehicles. It can also be applied to all types of battery-powered devices with higher power requirements.

\section{Acknowledgement}

The here presented work has been funded by the German Federal Ministry of Education and Research within the joint project "ViERforES-II - Virtual and Augmented Reality for Maximum Embedded System Safety, Security and Reliability" (01IM10002A) of the program IKT2020.

\section{References}

[1] Benecke, I.; Schmucker, U.: "Simulation statt Prüfstand" in Quality Engineering Vol 04.13, 2013, ISSN 1436-2457, pp. 40-42.

[2] Juhasz, T.; Schmucker, U.: "Automatic Model Conversion to Modelica for Dymolabased Mechatronic Simulation"; Proc. of 6th International Modelica Conference, 3rd-4th March, 2008, Bielefeld, Germany, Vol. 2, pp. 719-726.

[3] Schröder, D.: "Elektrische Antriebe - Regelung von Antriebssystemen"; Springer Verlag 2009; ISBN 978-3-540-89612-8

[4] FuelCon AG - http://www.fuelcon.com/

[5] MathWorks - http://www.mathworks.de/

[6] Böhme, T.; Kennel, M.; Schumann, M.; Winge, A.: "Automatisierte Erstellung domänenübergreifender Modelle und echtzeitfähige Kopplung von Simulation, Visualisierung und realen Steuerungen", HNI-
Verlagsschriftenreihe. Bd. 252. Paderborn, $2009-$ ISBN 978-3-939350-71-2, pp. $155-170$

[7] Dassault Systèmes - http://www.3ds.com/ 